\title{
GPDs and Orbital Angular Momentum
}

\author{
Matthias Burkardt ${ }^{* \dagger}$ \\ Department of Physics \\ New Mexico State University \\ Las Cruces, NM 88003 \\ U.S.A. Tel.: +1-575-6461928 \\ Fax: $+1-575-6461934$ \\ E-mail: burkardtenmsu.edu
}

Generalized Parton Distributions (GPDs) provide information on the distribution of quarks in impact paarmeter space. For transversely polarized nucleons, these impact parameter distributions are transversely distorted and this deviation from axial symmetry leads on average to a net transverse force from the spectators on the active quark in a DIS experiment. This force when acting along the whole trajectory of the active quark leads to transverse single-spin asymmetries. For a longitudinally polarized nucleon target, the transverse force implies a torque acting on the quark Orbital Angular Momentum (OAM). The resulting change in OAM as the quark leaves the target equals the difference between the Jaffe-Manohar and Ji OAMs. In the context of the scalar diquark model we demonstrate that the effect from that torque contributes at the same order as single-spin asymmetries

QCD Evolution 2016

May 30-June 03, 2016

National Institute for Subatomic Physics (Nikhef), Amsterdam

\footnotetext{
* Speaker.

${ }^{\dagger}$ This work was supported by the DOE under grant number DE-FG03-95ER40965.
} 


\section{Impact Parameter Dependent Parton Distributions}

Generalized Parton Distributions (GPDs) can be obtained from the same light-cone wave function overlap integrals that yield form factors, except that the momentum fraction $x$ of the active quark is not integrated over, i.e. GPDs can be understood as an $x$ decomposition of form factors. The 2-dimensional Fourier transform of the GPD $H_{q}(x, 0, t)$ yields the distribution $q\left(x, \mathbf{b}_{\perp}\right)$ of unpolarized quarks and target, in impact parameter space [1]

$$
q\left(x, \mathbf{b}_{\perp}\right)=\int \frac{d^{2} \Delta_{\perp}}{(2 \pi)^{2}} H_{q}\left(x, 0,-\Delta_{\perp}^{2}\right) e^{-i \mathbf{b}_{\perp} \cdot \Delta_{\perp}}
$$

with $\Delta_{\perp}=\mathbf{p}_{\perp}^{\prime}-\mathbf{p}_{\perp}$. For a transversely polarized target (e.g. polarized in the $+\hat{x}$-direction) the impact parameter dependent PDF $q_{+\hat{x}}\left(x, \mathbf{b}_{\perp}\right)$ is no longer axially symmetric and the transverse deformation is described by the gradient of the Fourier transform of the GPD $E_{q}(x, 0, t)$ [2]

$$
q_{+\hat{x}}\left(x, \mathbf{b}_{\perp}\right)=q\left(x, \mathbf{b}_{\perp}\right)-\frac{1}{2 M} \frac{\partial}{\partial b_{y}} \int \frac{d^{2} \Delta_{\perp}}{(2 \pi)^{2}} E_{q}\left(x, 0,-\Delta_{\perp}^{2}\right) e^{-i \mathbf{b}_{\perp} \cdot \Delta_{\perp}}
$$

$E_{q}(x, 0, t)$ and hence the details of this deformation are not very well known, but its $x$-integral, the Pauli form factor $F_{2}$, is. Eq. (1.2) allows to relate the average transverse deformation

$$
d_{y}^{q} \equiv \int d x \int d^{2} \mathbf{b}_{\perp} q\left(x, \mathbf{b}_{\perp}\right) b_{y}=\frac{1}{2 M} \int d x E_{q}(x, 0,0)=\frac{\kappa_{q}^{p}}{2 M}
$$

to the contribution from the corresponding quark flavor to the anomalous magnetic moment $\kappa_{u}^{p}=2 \kappa_{p}+\kappa_{n}=2 * 1.793-1.913=1.673$ and $\kappa_{d}^{p}=2 \kappa_{n}+\kappa_{p}=2 *(-1.913)+1.793=-2.033$. Since $\frac{1}{2 M} \approx 0.1 \mathrm{fm}$ this implies a very significant deformation $\left|d_{q}^{y}\right|=\mathscr{O}(0.2 \mathrm{fm})$ for both $u$ and $d$ quarks and in opposite directions.

For example, $u$ quarks in a proton contribute with a positive anomalous magnetic moment and $d$ quarks (after factoring out the negative $d$ quark charge) with a negative value. Eq. (1.2) thus implies that for a nucleon target polarized in the $+\hat{x}$ direction, the leading twist distribution of $u$ quarks is shifted in the $+\hat{y}$ direction while that of $d$ quarks is shifted in the $-\hat{y}$ direction.

GPDs provide information about the longitudinal momentum and the transverse position of partons. This is reminiscent of angular momentum, which requires position and momentum information in orthogonal directions. In a state that is an eigenstate under rotations about the $\hat{x}$ axis, the expectation value of the momentum density in the $\hat{y}$ direction weighted by the position in the $\hat{z}$ direction is up to a sign numerically equal to the expectation value of the momentum density in the $\hat{z}$ direction weighted by the position in the $\hat{y}$ direction. This is why the transverse deformations of quark distributions described by GPDs can tell us about the contribution from that quark flavor to the nucleon spin. Given that $E(x, 0, t)$ describes the transverse deformation of quark distributions in a transversely polarized target it is thus not surprising the the $x$-moment of $E(x, 0, t)$ plays a role in the angular momentum sum rule. However, $E(x, 0, t)$ describes only the deformation relative to the center of momentum of the entire nucleon. It turns out that a delocalized nucleon wave packed for a transversely polarized nucleon that is centered at the origin when boosted to the infinite momentum frame is has a center of momentum that is shifted sideways by half a Compton wavelength. As 
a result there is an additional contribution involving the momentum fraction carried by that quark flavor [3]

$$
J_{q}=\frac{1}{2} \int_{0}^{1} d x x\left[H^{q}(x, 0,0)+E^{q}(x, 0,0)\right],
$$

which was first derived in Ref. [4].

\section{Angular Momentum Decompositions}

Unfortunately, there are additional complications due to issues related to the definition of OAM. To illustrate that there may be issues with how OAM is defined, let us start from a definition of photon total angular momentum based on the Poynting vector. First one rewrites the magnetic field in terms of the vector potential.

Since the famous EMC experiments revealed that only a small fraction of the nucleon spin is due to quark spins[5], there has been a great interest in 'solving the spin puzzle', i.e. in decomposing the nucleon spin into contributions from quark/gluon spin and orbital degrees of freedom. In this effort, the Ji decomposition[4]

$$
\frac{1}{2}=\frac{1}{2} \sum_{q} \Delta q+\sum_{q} L_{q}^{z}+J_{g}^{z}
$$

appears to be very useful: through GPDs, not only the quark spin contributions $\Delta q$ but also the quark total angular momenta $J_{q} \equiv \frac{1}{2} \Delta q+L_{q}^{z}$ (and by subtracting the spin piece also the the quark orbital angular momenta $L_{q}^{z}$ ) entering this decomposition can be accessed experimentally. In the Ji decomposition (2.1) the quark OAM is defined as the expectation value

$$
L_{q}^{z}=\int d^{3} r\left\langle P S\left|q^{\dagger}\left(\vec{r} \times \frac{1}{i} \vec{D}\right)^{z} q\right| P S\right\rangle /\langle P S \mid P S\rangle
$$

in a nucleon state polarized in the $+\hat{z}$ direction. Here $\vec{D}=\vec{\partial}-i g \vec{A}$ is the gauge-covariant derivative. The main advantages of this decomposition are that each term can be expressed as the expectation value of a manifestly gauge invariant local operator and that the quark total angular momentum $J^{q}=\frac{1}{2} \Delta q+L^{q}$ can be related to GPDs[4] and is thus accessible in deeply virtual Compton scattering and deeply virtual meson production and can also be calculated in lattice gauge theory.

Jaffe and Manohar have proposed an alternative decomposition of the nucleon spin, which does have a partonic interpretation, and in which also two terms, $\frac{1}{2} \Delta q$ and $\Delta G$, are experimentally accessible [6]

$$
\frac{1}{2}=\frac{1}{2} \sum_{q} \Delta q+\sum_{q} \mathscr{L}^{q}+\Delta G+\mathscr{L}^{g}
$$

In this decomposition the quark OAM is defined as

$$
\mathscr{L}^{q} \equiv \int d^{3} r\left\langle P S\left|q_{+}^{\dagger}\left(\vec{r} \times \frac{1}{i} \vec{\partial}\right)^{z} q_{+}\right| P S\right\rangle /\langle P S \mid P S\rangle,
$$

where light-cone gauge $A^{+}=0$ is implied. Although Eq. (2.4) is not manifestly gauge invariant as written, gauge invariant extensions can be defined $[9,10]$. Indeed, manifestly gauge invariant 
definitions for each of the terms in Eq. (2.3) exist, which, with the exception of $\Delta q$, involve matrix elements of nonlocal operators. In light-cone gauge those nonlocal operators reduce to a local operator, such as Eq. (2.4).

$$
\begin{aligned}
\vec{J}_{\gamma} & =\int d^{3} r \vec{r} \times(\vec{E} \times \vec{B})=\int d^{3} r \vec{r} \times[\vec{E} \times(\vec{\nabla} \times \vec{A})] \\
& =\int d^{3} r\left[E^{j}(\vec{r} \times \vec{\nabla}) A^{j}-\vec{r} \times(\vec{E} \cdot \vec{\nabla}) \vec{A}\right] \\
& =\int d^{3} r\left[E^{j}(\vec{r} \times \vec{\nabla}) A^{j}+(\vec{r} \times \vec{A}) \vec{\nabla} \cdot \vec{E}+\vec{E} \times \vec{A}\right] \\
& =\int d^{3} r\left[E^{j}(\vec{r} \times \vec{\nabla}) A^{j}+(\vec{r} \times \vec{A}) e \psi^{\dagger} \psi+\vec{E} \times \vec{A}\right]
\end{aligned}
$$

Upon simplifying the double cross product inside the square bracket, one finds a term that involves the fields $(\vec{A})$ as well as their canonical momenta $(\vec{E})$ sandwiching $\vec{r} \times \vec{\nabla}$. That term has a physical interpretation as orbital angular momentum for the photons. In the next step, one can integrate by parts (drop surface terms) the remaining terms. This results in two contributions: one where the gradient acts on the position vector, resulting in a term $\vec{E} \times \vec{A}$ that acts only on the internal degrees of freedom and thus has an interpretation as the photon spin. The other term that arises from integration by ports involves the divergence of the electric field which, using the equations of motion, can be replaced by the charge density. This results in a term that is up to a sign identical to the term involving the vector potential in the local manifestly gauge invariant definition of the quark OAM. In summary, what has happened is that integrating by parts and using the equations of motion we transformed the photon total angular momentum into photon spin plus photon orbital angular momentum plus a term that cancels the term in the electron (local \& manifestly gauge invariant) OAM $\psi^{\dagger} \vec{r} \times(\vec{p}-e \vec{A}) \psi$

- replace $2^{\text {nd }}$ term (eq. of motion $\vec{\nabla} \cdot \vec{E}=e j^{0}=e \psi^{\dagger} \psi$ ), yielding

$$
\vec{J}_{\gamma}=\int d^{3} r\left[\psi^{\dagger} \vec{r} \times e \vec{A} \psi+E^{j}(\vec{x} \times \vec{\nabla}) A^{j}+\vec{E} \times \vec{A}\right]
$$

- $\psi^{\dagger} \vec{r} \times e \vec{A} \psi$ cancels similar term in electron OAM $\psi^{\dagger} \vec{r} \times(\vec{p}-e \vec{A}) \psi$

$\hookrightarrow$ decomposing $\vec{J}_{\gamma}$ into spin and orbital also shuffles angular momentum from photons to electrons!

\section{OAM from Wigner Distributions}

Given the fact that different spin decompositions and corresponding definitions for quark OAM are possible raises the question about the physical interpretation of those differences. In this effort significant progress has been made based on 5-dimensional Wigner distributions.

Wigner distributions can be defined as off forward matrix elements of non-local correlation functions $[7,8,9]$ with $P^{+}=P^{+\prime}, P_{\perp}=-P_{\perp}^{\prime}=\frac{q_{\perp}}{2}$

$$
W^{\mathscr{U}}\left(x, \vec{b}_{\perp}, \vec{k}_{\perp}\right) \equiv \int \frac{d^{2} \vec{q}_{\perp}}{(2 \pi)^{2}} \int \frac{d^{2} \xi_{\perp} d \xi^{-}}{(2 \pi)^{3}} e^{-i \vec{q}_{\perp} \cdot \vec{b}_{\perp}} e^{i\left(x P^{+} \xi^{-}-\vec{k}_{\perp} \cdot \vec{\xi}_{\perp}\right)}\left\langle P^{\prime} S^{\prime}\left|\bar{q}(0) \Gamma \mathscr{U}_{0 \xi} q(\xi)\right| P S\right\rangle .
$$


Throughout this paper, we will chose $\vec{S}=\vec{S}^{\prime}=\hat{\vec{z}}$. Furthermore, we will focus on the 'good' component by selecting $\Gamma=\gamma^{+}$. To ensure manifest gauge invariance, a Wilson line gauge link $\mathscr{U}_{0 \xi}$ connecting the quark field operators at position 0 and $\xi$ is included. The issue of choice of path for the Wilson line will be addressed below.

In terms of Wigner distributions, TMDs and OAM can be defined as [9]

$$
\begin{aligned}
f\left(x, \vec{k}_{\perp}\right) & =\int d x d^{2} \vec{b}_{\perp} d^{2} \vec{k}_{\perp} \vec{k}_{\perp} W^{\mathscr{U}}\left(x, \vec{b}_{\perp}, \vec{k}_{\perp}\right) \\
L_{\mathscr{U}} & =\int d x d^{2} \vec{b}_{\perp} d^{2} \vec{k}_{\perp}\left(\vec{b}_{\perp} \times \vec{k}_{\perp}\right)^{z} W^{\mathscr{U}}\left(x, \vec{b}_{\perp}, \vec{k}_{\perp}\right) .
\end{aligned}
$$

No issues with Heisenberg's uncertainty principle arise here: only perpendicular combinations of position $\vec{b}_{\perp}$ and momentum $\vec{k}_{\perp}$ are needed simultaneously in order to evaluate the integral for $L_{\mathscr{U}}$.

A straight line connecting 0 and $\xi$ for the Wilson line in $\mathscr{U}_{0 \xi}$ results in [7]

$$
L_{\text {straight }}^{q}=L_{J i}^{q} \text {. }
$$

However, depending on the context, other choices for the path in the Wilson link $\mathscr{U}$ should be made. Indeed for TMDs probed in SIDIS the path should be taken to be a straight line to $x^{-}=\infty$ along (or, for regularization purposes, very close to) the light-cone. This particular choice ensures proper inclusion of the FSI experienced by the struck quark as it leaves the nucleon along a nearly light-like trajectory in the Bjorken limit. However, a Wilson line to $\xi^{-}=\infty$, for fixed $\vec{\xi}_{\perp}$ is not yet sufficient to render Wigner distributions manifestly gauge invariant, but a link at $\xi^{-}=\infty$ must be included to ensure manifest gauge invariance. While the latter may be unimportant in some gauges, it is crucial in light-cone gauge for the description of TMDs relevant for SIDIS.

Let $\mathscr{U}_{0 \xi}^{+L C}$ be the Wilson path ordered exponential obtained by first taking a Wilson line from $\left(0^{-}, \overrightarrow{0}_{\perp}\right)$ to $\left(\infty, \overrightarrow{0}_{\perp}\right)$, then to $\left(\infty, \vec{\xi}_{\perp}\right)$, and then to $\left(\xi^{-}, \vec{\xi}_{\perp}\right)$, with each segment being a straight line (Fig. 1) [10]. The shape of the segment at $\infty$ is irrelevant as the gauge field is pure gauge there, but

Figure 1: Illustration of the path for the Wilson line gauge link $\mathscr{U}_{0 \xi}^{+L C}$ entering $W^{+L C}$

it is still necessary to include a connection at $\infty$ and for simplicity we pick a straight line. Likewise, with a similar 'staple' to $-\infty$ we define the Wilson path ordered exponential $\mathscr{U}_{0 \xi}^{-L C}$, and using those light-like gauge links, we define

$$
W^{ \pm L C}\left(x, \vec{b}_{\perp}, \vec{k}_{\perp}\right) \equiv \int \frac{d^{2} \vec{q}_{\perp}}{(2 \pi)^{2}} \int \frac{d^{2} \xi_{\perp} d \xi^{-}}{(2 \pi)^{3}} e^{-i \vec{q}_{\perp} \cdot \vec{b}_{\perp}} e^{i\left(x P^{+} \xi^{-}-\vec{k}_{\perp} \vec{\xi}_{\perp}\right)}\left\langle P^{\prime} S^{\prime}\left|\bar{q}(0) \Gamma \mathscr{U}_{0 \xi}^{ \pm L C} q(\xi)\right| P S\right\rangle .
$$

This definition for $W^{+L C}$ the same as that in Ref. [10] and similar to that of $W_{L C}$ in Ref. [7] (the link segment at $\xi^{-}=\infty$ was not included in the definition of $W_{L C}$ ).

In light-cone gauge $A^{+}=0$, only the segment at $\xi^{-}= \pm \infty$ contributes and the OAM looks similar to the local manifestly gauge invariant expression, except

$$
\vec{r} \times \vec{A}(\vec{r}) \longrightarrow \vec{r} \times \vec{A}\left(r^{-}= \pm \infty, \mathbf{r}_{\perp}\right) .
$$




\section{Bashinsky-Jaffe Orbital Angular Momentum}

Imposing the condition $A^{+}=0$ does not completely fix the gauge as one can still apply residual gauge transformations $A^{\mu}(x) \longrightarrow A^{\mu}(x)+\partial^{\mu} \phi\left(x_{\perp}\right)$. In the context of quark OAM there are (at least) two options to deal with this situation. One option is to attach light-cone staple-shaped Wilson line gauge links to render the definition of $\mathscr{L}^{q}$ manifestly gauge invariant. In the following we will denote this option as $\mathscr{L}_{ \pm}$for future and past pointing lightcone staples respectively. PT invariance implies that $\mathscr{L}_{-}=\mathscr{L}_{+}$[10]. In light-cone gauge only the gauge link segment at lightcone infinity contributes resulting in a derivative $i \partial^{\perp}-g A^{\perp}\left( \pm \infty, x_{\perp}\right)$.

Bashinsky and Jaffe addressed the issue of residual gauge invariance by introducing a gaugecovariant derivative that contains the 'zero-mode' of $\vec{A}_{\perp}$

$$
\overrightarrow{\mathscr{A}}_{\perp}\left(\mathbf{x}_{\perp}\right) \equiv \frac{\int d x^{-} \vec{A}_{\perp}\left(x^{-}, \vec{x}_{\perp}\right)}{\int d x^{-}}
$$

into the definition of quark OAM, which renders $\mathscr{L}_{J B}^{q}$ invariant under residual gauge transformations. Since the gluon field strength tensor should vanish at $x^{-}= \pm \infty, \vec{A}_{\perp}\left(x^{-}, \vec{x}_{\perp}\right)$ should approach a limiting value at $x^{-}= \pm \infty$ and hence $\overrightarrow{\mathscr{A}}_{\perp}\left(\mathbf{x}_{\perp}\right)$ should be dominated by these values, which results in

$$
\mathscr{L}_{J B}^{q}=\frac{1}{2}\left(\mathscr{L}_{+}^{q}+\mathscr{L}_{-}^{q}\right)=\mathscr{L}_{+}^{q}=\mathscr{L}_{-}^{q}
$$

In the last steps PT invariance was used. This demonstrates that $\mathscr{L}_{J B}^{q}$ is identical to definitions using light-cone staples.

From PT invariance one finds that $\mathscr{L}_{+}^{q}=\mathscr{L}_{-}^{q}$ [10]. In the Bashinsky-Jaffe definition of OAM $\mathscr{L}_{B J}^{q}[12]$, the vector potential in the gauge covariant derivative is replaced by

$$
\frac{\int_{-\infty}^{\infty} d x^{-} A_{\perp}\left(r^{-}, \mathbf{r}_{\perp}\right)}{\int_{-\infty}^{\infty} d x^{-}}=\frac{1}{2}\left[A_{\perp}\left(r^{-}=\infty, \mathbf{r}_{\perp}\right)+A_{\perp}\left(r^{-}=\infty, \mathbf{r}_{\perp}\right)\right]
$$

and is thus equivalent to the light-cone-staple definition

$$
\mathscr{L}_{B J}^{q}=\frac{1}{2}\left(\mathscr{L}_{+}^{q}+\mathscr{L}_{-}^{q}\right)=\mathscr{L}_{+}^{q}=\mathscr{L}_{-}^{q}
$$

Imposing $A^{+}=0$ does not completely fix the gauge as one can still make $r^{-}$-independent gauge transformations. If one fixes this residual gauge invariant by imposing anti-symmetric boundary conditions $A_{\perp}\left(r^{-}=-\infty, \mathbf{r}_{\perp}\right)=-A_{\perp}\left(r^{-}=-\infty, \mathbf{r}_{\perp}\right)$ the vector potential at $r^{-}= \pm \infty$ cancels out in $\mathscr{L}_{+}^{q}+\mathscr{L}_{-}^{q}$ and therefore, with the understanding of anti-symmetric boundary conditions at $r^{-}= \pm \infty$ the Jaffe-Manohar OAM becomes also identical to $\mathscr{L}_{ \pm}^{q}$. This observation is crucial for understanding the difference between the Ji vs. Jaffe-Manohar OAM, which in light-cone gauge ${ }^{1}$ involves only the replacement $A_{\perp}^{i}(\vec{r}) \longrightarrow A_{\perp}^{i}\left(r^{-}= \pm \infty, \mathbf{r}_{\perp}\right)$. Using

$$
A_{\perp}^{i}\left(r^{-}=\infty, \mathbf{r}_{\perp}\right)-A_{\perp}^{i}\left(r^{-}, \mathbf{r}_{\perp}\right)=\int_{r^{-}}^{\infty} d z^{-} \partial_{-} A_{\perp}^{i}\left(z^{-}, \vec{r}_{\perp}\right)=\int_{r^{-}}^{\infty} d z^{-} G^{+i}\left(z^{-}, \vec{r}_{\perp}\right)
$$

\footnotetext{
${ }^{1}$ As $L^{q}$ involves a manifestly gauge invariant local operator, it can be evaluated in any gauge.
} 
where $G^{+\perp}=\partial_{-} A^{\perp}$ is the gluon field strength tensor in $A^{+}=0$ gauge. Note that

$$
-\sqrt{2} g G^{+y} \equiv-g G^{0 y}-g G^{z y}=g\left(E^{y}-B^{x}\right)=g(\vec{E}+\vec{v} \times \vec{B})^{y}
$$

yields the $\hat{y}$ component of the color Lorentz force acting on a particle that moves with the velocity of light in the $-\hat{z}$ direction $(\vec{v}=(0,0,-1))$ - which is the direction of the momentum transfer in DIS $[?, 13]$. Thus the difference between the Jaffe-Manohar and $\mathrm{Ji}^{2} \mathrm{OAMs}$

$$
\mathscr{L}^{q}-L^{q}=-g \int d^{3} x\left\langle P, S\left|\bar{q}(\vec{x}) \gamma^{+}\left[\vec{x} \times \int_{x^{-}}^{\infty} d r^{-} F^{+\perp}\left(r^{-}, \mathbf{x}_{\perp}\right)\right]^{z} q(\vec{x})\right| P, S\right\rangle /\langle P S \mid P S\rangle
$$

has the semiclassical interpretation of the change in OAM due to the torque from the FSI as the quark leaves the target:[11] while $L^{q}$ represents the local and manifestly gauge invariant OAM of the quark before it has been struck by the $\gamma^{*}, \mathscr{L}^{q}$ represents the gauge invariant OAM after it has left the nucleon and moved to $r^{-}=\infty$.

\section{Intuitive Picture for the Torque from Final State Interactions}

In order to estimate the effect from the final state interactions on the quark OAM we first consider the effect on a positron moving through the magnetic dipole field of an electron, which is polarized in the $+\hat{z}$ direction. This should be the most simple analogy to a proton polarized in the $+\hat{z}$ direction because more quarks are polarized in the same direction as the nucleon spin and the color-electric force between the active quark and the spectators is attractive. As illustrated in Fig.

Figure 2: Illustration of the torque acting on a positron moving in the $-\hat{z}$ direction through a magnetic dipole field caused by the magnetic moment of an electron polarized in the $+\hat{z}$ direction. a.) side view; b.) top view. In this example the $\hat{z}$ component of the torque is negative as the positron leaves the bound state.

2 the magnetic FSI leads to a negative torque. Since the example was chosen such that the signs of

\footnotetext{
${ }^{2}$ Here we replaced $\gamma^{0} \rightarrow \gamma^{+}$in $L^{q}$ as discussed in Ref. [14].
} 
polarization and forces are the same as in the nucleon this implies that the color-magnetic torque acting on quarks ejected from the proton are negative as well.

The dipole example also illustrates why the torque vanishes immediately after the absorption of the virtual photon: when comparing the $\perp$ magnetix field (for fixed $\perp$ position) at positions at the 'front' and 'back' side of the nucleon are equal and opposite, and therefore the torque at the original position of the active quark averages to zero

$$
\left\langle P, S\left|\bar{q}(0)\left[x F^{+y}(0)-y F^{+x}(0)\right] q(0)\right| P, S\right\rangle=0 .
$$

Note that this cancellation is a consequence of averaging over all possible initial positions of the active quark. For a specific initial position the initial torque will in general be nonzero. While we used here an intuitive picture to motivate this result, it can also be shown to be a rigorous consequence of PT invariance.

The same example also illustrates the nonzero net effect from the final state interaction torque: although there is some partial cancelation from the 'front' and 'back' of the nucleon, after integrating along the trajectory of the ejected quark, the torque from the 'back' impacts more quarks than that from the 'front' resulting in a nonzero ensemble average.

\section{Torque in Spectator Models}

${ }^{3}$ Given the fact that the Jaffe-Manohar and Ji definitions of quark orbital angular momentum are differ by the potential angular momentum raises the question as how significant that difference actually is. In Ref. [?] it was shown that to $\mathscr{O}(\alpha)$ in QED $L_{J i}=\mathscr{L}_{J M}$. This corrects an earlier result [14] where the contribution from states with longitudinally polarized Pauli-Villars photons had been omitted.

In order to assess the significance of FSI effects for quark OAM we thus considered the effects of the vector potential in the scalar diquark model. While we do not consider this model a good approximation for QCD, it has been very useful in several respects:

Most importantly, the model allows for a fully Lorentz invariant calculation of 'nucleon' matrix elements - which is not the case for almost all other models for nucleon structure. Since Lorentz invariance is heavily used for example in the derivation of Eq. ??, this is a crucial aspect of the model. We also note that this was the first model that clearly illustrated the role of FSI and the Sivers in SIDIS and DY [16].

In this model we directly evaluated the potential angular momentum by calculating nonforward matrix elements of $\bar{q} \gamma^{+} A^{y} q$ with momentum transfer $\Delta_{\perp}$. Upon taking $\left.\frac{d}{d \Delta^{x}}\right|_{\Delta=0}$ of these matrix elements one obtains the forward matrix element of $x \bar{q} \gamma^{+} A^{y} q$. The vector potential $A^{y}$ in this matrix element is evaluated perturbatively using Feynman rules. This results in an 'exchange' diagram where the photon originates from the spectators (Fig. 3) as well as a diagram where the photon originates from the active quark line.

We found that a nonzero potential angular momentum arises at the same order as transverse single-spin asymmetries [16], i.e. one photon/gluon exchange. Numerically, one finds at lowest

\footnotetext{
${ }^{3}$ In collaboration with C.Lorcé
} 
nontrivial order that the average transverse momentum and the (dimensionless) potential angular momentum differ only by a 'quark'/'nucleon' mass factor times a factor of $\mathscr{O}(1)$

$$
\left\langle k_{\perp}^{q}\right\rangle=\frac{3 m_{q}+M}{12} \pi\left\langle\bar{q} \gamma^{+}(\vec{r} \times \vec{A})^{z} q\right\rangle
$$

which confirms that the potential angular momentum is as important as transverse single-spin asymmetries.

For the example of the scalar diquark model we found that $L_{p o t} \equiv L_{J M}-L_{J i}$ is positive. This result can be understood as follows: in the scalar diquark model both the OAM of the 'quark' and that of the diquark are positively correlated with the nucleon spin. Moreover, when the active quark and the diquark are orbiting their common center of momentum their transverse momenta are always pointing in opposite directions. For example, when the 'nucleon' spin is pointing up, the magnetic field from the orbital motion of the diquark (negative charge) at the position of the active quark (positive charge) is always pointing 'out' above the plane of orbit and 'in' below it. Thus regardless whether the active quark is ejected 'upward' or 'downward', the Lorentz force due to the transverse component of the magnetic field of the spectator will be directed in the direction of motion before being ejected, i.e. the change in OAM is positive.

Figure 3: Please write your figure caption here

Figure 4: Please write your figure caption here

7. ...

\section{References}

[1] M. Burkardt, Phys.Rev. D62 (2000) 071503, Erratum: Phys.Rev. D66 (2002) 119903.

[2] M. Burkardt, Int.J.Mod.Phys. A18 (2003) 173-208.

[3] M. Burkardt, hep-ph/0105324.

[4] X. Ji, Phys. Rev. Lett. 78 (1997) 610.

[5] J. Ashman et al (EMC), Phys. Lett. B 206 (1988) 364.

[6] R.L. Jaffe and A. Manohar, Nucl. Phys. B 337 (1990) 509.

[7] A.V. Belitsky, X. Ji, and F. Yuan, Phys. Rev. D 69 (2004) 074014.

[8] S. Meissner, A. Metz, and M. Schlegel, AIP Conf.Proc. 1149 (2009) 539; JHEP 0908 (2009) 056.

[9] C. Lorcé and B. Pasquini, Phys. Rev. D 84 (2011) 014015.

[10] Y. Hatta, Gluon Polarization in the Nucleon Demystified, Phys. Rev. D 84, 041701 (2011); Phys. Lett. B 708 (2012) 186; Phys. Rev. Lett. 67 (1991) 2264.

[11] M. Burkardt, Phys. Rev. D 88 (2013) 1.

[12] S. Bashinsky and R.L. Jaffe, Nucl. Phys. B536 (1998) 303. 
[13] M. Burkardt, Phys. Rev. D 88 (2013) 114502.

[14] M. Burkardt and H. BC, Phys. Rev. D 79 (2009) 071501.

[15] X. Ji et al., Phys.Rev. D93 (2016) 054013.

[16] S.J. Brodsky, D.-S. Hwang, and I. Schmidt, Phys.Lett. B530 (2002) 99. 